\title{
An Organization's Extended (Soft) Competencies Model
}

\author{
João Rosas ${ }^{1}$, Patrícia Macedo ${ }^{1,2}$, and Luis M. Camarinha-Matos ${ }^{1}$ \\ ${ }^{1}$ New University of Lisbon, Faculty of Sciences and Technology, Portugal \\ ${ }^{2}$ Polytechnic Institute of Setubal, Portugal \\ jrosas@uninova.pt, pmacedo@est.ips.pt, cam@uninova.pt
}

\begin{abstract}
One of the steps usually undertaken in partnerships formation is the assessment of organizations' competencies. Typically considered competencies of a functional or technical nature, which provide specific outcomes can be considered as hard competencies. Yet, the very act of collaboration has its specific requirements, for which the involved organizations must be apt to exercise other type of competencies that affect their own performance and the partnership success. These competencies are more of a behavioral nature, and can be named as soft-competencies. This research aims at addressing the effects of the soft competencies on the performance of the hard ones. An extended competencies model is thus proposed, allowing the construction of adjusted competencies profiles, in which the competency levels are adjusted dynamically according to the requirements of collaboration opportunities.
\end{abstract}

Keywords: Soft Competencies, Hard Competencies, Collaboration.

\section{Introduction}

In partnership formation, a competencies' assessment is usually done in order to determine the suitability of potential partners and to establish which activities should be assigned to which organizations, in an effort to build the best network with an increased chance of achieving the desired goals. This competencies' assessment inform about the organizations capability to perform a number of related tasks, activities or processes. However, it usually happens that these competencies are typically tuned for working as a single entity in a regular market-like environment. Although accurate for such environment, considering just these competencies might not be enough in a collaboration context. This comes from the fact that a collaboration process has its specific requirements, to which organizations must be apt to comply, or otherwise their performance inside the partnership is affected. In order to comply with these collaboration-related requirements, it is necessary that organizations own other type of competencies. While the former competencies are more functional and technical, the later are of a more behavioral nature. As such, we can refer to hard and soft competencies.

This research aims at identifying the performance effects of the soft competencies on the hard ones, within a collaboration context. The duality between soft and hard competencies is observed from a behavioral perspective, which also considers the very values of an organization, its traits, and the activities performed at a more functional and technical level. A modeling approach is proposed combining both hard and 
soft competencies. The assumption is that considering these aspects in the partners' competencies assessment provides a broader and more accurate perception of partners' capabilities, and that such vision of capabilities allows an improved assignment of roles and activities inside networks. This assignment in turn leads to the formation of more effective consortiums.

In order to achieve these aims, a number of concepts are introduced, such as the Extended Competencies Model, the Adjusted Competency Model, and Adjusted Competency Level. An example illustrating how to use the proposed approach is presented afterwards.

\section{Literature Review}

The Competencies profile subject has been studied during the last decades, using diverse approaches and for distinct purposes. For instance, in Human Resource Management research, competencies are studied from the point of view of Job Competencies in which they are considered as technical skills to perform job activities. In the last decades, some authors initiated a new approach to competency management, introducing the importance of some behavioral characteristics for the proficiency of professional careers [1]. The term "soft competencies" was defined as "personal behaviors or attitudes". Diverse authors defended that soft-competencies are complementary to technical competencies, and that they are of great importance in human resource management [2] [3].

In [4] a model for competency management in organizations called "Core competency notion" is proposed in order to support strategic planning and provide means for achieving better synergies among the various organization's business units. Another contribution to this issue was provided by [5] proposing an extension of the core competency notion, where the concepts of resource and capabilities are included in the core-competency model. He proposed also, a method based on the discussion of eight structured questions that will help managers to identify the company's core competencies and capabilities in a systematic and methodical way.

In recent years, the collaborative networks community developed some work related to competency management in a collaborative context. Molina and Flores [6] proposed a core-competency model for the manufacturing clusters. The basic idea of this model is to match the tasks defined for a new Virtual Organization against the constituent skills provided by the cluster of organizations. Another model for competency analysis in collaborative context, called s-a-r-C model, was introduced by Boucher and Leburean [7]. This model supports the idea that competencies usually increase in networked organizations as a consequence of the interaction between tasks, human resources and material capabilities. Odenthal and Peters [8] further developed the concept of competency profiles in collaborative environments, proposing a method to generate target competency profiles in a Virtual Enterprise. These target profiles are based on the allocation of competencies to activities and where each set of activities correspond to a specific task. Recently Ermilova and Afsarmanesh [9] developed a competency model specific to competencies management in Virtual Organizations Breeding Environments (VBEs). This model is called the "4C-model" and 
considers four fundamental components of competency: "Capability", "Capacity", "Cost" and "Conspicuity".

Although the soft competencies concept has been addressed in related fields, our perception led us to conclude that, in spite of the potential value and benefits it could bring, the subject of soft versus hard competencies has so far received little attention in collaborative networks.

\section{The Extended Competencies Model}

Model specification. In order to establish an approach and adequate analysis, it is important to mention that the aim of this research is not to obtain a complete and detailed competencies model, but as mentioned before, to address the soft competencies performance effects. As such, the adopted approach is to define an abstract model, maintaining the compliance with existing models, but allowing the integration of the findings from this research.

The meaning of competency, according to the Cambridge dictionary, corresponds basically to the ability to do something successfully or efficiently [10]. In a similar way, an organization that is considered competent on a certain domain has got the necessary ability, knowledge, and skills to perform the corresponding tasks towards achieving specified goals [1].

Competencies can be either of hard or soft nature. In order to establish an adequate framework, this dual notion of competencies must be considered inside a behavioral space, in which the relevant organization's behaviors can be characterized. This space, as illustrated in Fig. 1, starts from the very values of an organization and spans to the activities and tasks performed at a technical and functional level.

\begin{tabular}{|c|c|c|c|c|c|}
\hline \multicolumn{6}{|c|}{ Organizational Behavior } \\
\hline $\begin{array}{l}\text { Values } \\
\text { System }\end{array}$ & $\begin{array}{c}\text { Organization } \\
\text { Traits }\end{array}$ & $\begin{array}{c}\text { Soft } \\
\text { Competencies }\end{array}$ & $\begin{array}{c}\text { Hard } \\
\text { Competencies }\end{array}$ & Activities & $\begin{array}{c}\text { Task } \\
\text { Processes }\end{array}$ \\
\hline $\begin{array}{l}\text { abstract } \\
\text { behavior }\end{array}$ & & behav & nature & & $\begin{array}{l}\text { targeted } \\
\text { behavior }\end{array}$ \\
\hline
\end{tabular}

Fig. 1. Organization's competencies in a continuous behavioral space

Value systems are related both to the purposes of an organization and its roles inside society. The behaviors developed by organizations should, in principle, be in accordance with their values, and their influences are propagated to the lower technical and functional behavioral levels ${ }^{1}$. Principles of ethics and code of conduct are also of great importance in collaborative networks $[11,12]$. Next to the values, there are organization traits, which are also values, but with a more concrete connotation to organizations' behaviors. These traits can be perceived from the behavioral patterns that arise from the behaviors performed by organizations. For instance, an organization may be classified as reliable because it has performed in a very reliable way, and

\footnotetext{
${ }^{1}$ One can imagine a 'green' enterprise adopting renewable energies and performing recycling in its manufacturing system.
} 
can be considered friendly if it reveals friendly attitudes. This organization could therefore be characterized as owning the traits of reliability and friendliness.

Soft competencies come next to traits. This "proximity" is related to the fact that soft competencies are more behavioral, as opposite to the hard ones, which are connected to a more functional level. The ability to develop soft competencies is in accordance to the mentioned organizations traits. For instance, if an organization is capable of building consensus on a conflicting situation, which is an example of a soft competency, that might be due to its empathy and openness, which are examples of traits. Going further through the considered behavioral space, we can find the hard competencies concept. Such type of competencies is associated to the specific skills, functions, activities, and knowledge, used to achieve specific goals and outcomes, establishing the association to the mentioned more technical and functional nature.

These concepts can be represented using a formal notation, starting from traits and covering all the concepts mapped in the mentioned behavioral space. For subsequent modeling, let us consider the set of organizations $O=\left\{o_{1}, o_{2}, o_{3}, \ldots\right\}$.

Definition 1 (Organization's Behaviors). The organization's behaviors can be represented by the set $B=\left\{b_{1}, b_{2}, b_{3}, \ldots\right\}$, in which each behavior $b_{\text {i }}$ represents a way an organization acts or conducts itself and toward others, or the way it behaves in response to a particular event, internal or external stimuli, or situation.

Definition 2 (Organization's Traits). A set $T=\left\{t_{1}, t_{2}, t_{3}, \ldots\right\}$ where each trait $t_{\mathrm{i}}$ represents a relatively stable predisposition to act in a certain way, and which can be used to describe or characterize the behavior of organizations (e.g. reliability, openness, and adaptability).

Definition 3 (Organization's Soft Competencies). A set $S C=\left\{s c_{1}, s c_{2}, s c_{3}, \ldots\right\}$, in which each element $s c_{\mathrm{i}}$ can be abstractly understood as a general aptitude to perform a behavior (e.g. the ability to exchange knowledge), which is beneficial for the achievement of the outcomes associated to the performance of a hard competency.

The following definition introduces the concept of hard competence, which is built upon the $4 C$ competence model described in [9].

Definition 4 (Organization's Hard Competency). Abstractly understood as an aptitude to act towards achieving specified outcomes or goals. It represents an organization's capability to run activities, tasks or processes, which allows achieving concrete outcomes or goals. Assuming a set of given hard competences $H C=\left\{h c_{1}, h c_{2}, h c_{3} \ldots\right\}$, each of its elements can be defined as a tuple $h c_{\mathrm{i}}=\left(C_{i, 1}, C_{i, 2}, C_{i, 3}, C_{i, 4}\right)$, such that (taking the index ' $i$ ' out):

- $C_{1}$ represents the competence capability information in terms of the processes and activities an organization can perform.

- $C_{2}$ represents the competence capacity information, used to represent the free capacity of related resources, and to specify quantitative values of capabilities, such as a production rate (e.g. units/day).

- $C_{3}$ represents cost information of products/services that are related to the organization's capability $C_{1}$. 
- $C_{4}$ represents conspicuity information, which is used as a mean to validate and certify the organizations' capabilities. This conspicuity information can take the form of certifications, licenses, or recommendation letters.

The following definition introduces the extended competency model concept, which results from the combination of an organization's traits, observed behaviors, and both the hard and soft competencies specified in the previous definitions.

Definition 5 (Organization's Extended Competencies model). Can be specified as a tuple $\mathrm{SCP}=\left(\mathrm{o}_{\mathrm{k}}, O T, O B, H C L, S C L\right)$, where:

- $o_{\mathrm{k}}$ - represents a given organization, such that $o_{\mathrm{k}} \in O$.

- $O T=\left\{\left(t_{\mathrm{i}}, v_{\mathrm{i}}\right) \mid t_{\mathrm{i}} \in T, v_{\mathrm{i}} \in\{\right.$ low, average, high $\left.\}\right\}$ are the organization's traits.

- $O B=\left\{\left(b_{i}, v_{i}\right) \mid b_{i} \in B, v_{i} \in\{\right.$ low, average, high $\left.\}\right\}$ are observed organization's behaviors, associated to previously observed soft competencies manifestations.

- $H C L=\left\{\left(h c_{\mathrm{i}}, l_{\mathrm{i}}\right) \mid h c_{\mathrm{i}} \in H C, l_{\mathrm{i}} \in[0,100]\right\}$. The value of $l_{\mathrm{i}}$ represents the $h c_{i}$ competency level. HC is the set of hard competencies (as in Definition 4).

- $S C L=\left\{\left(s c_{\mathrm{i}}, l_{\mathrm{i}}\right) \mid s c_{\mathrm{i}} \in S C, l_{\mathrm{i}} \in\{\right.$ low, average, high $\left.\}\right\}$. The level $l_{\mathrm{i}}$ indicates the level of the soft competency $s c_{\mathrm{i}}$, for the organization $o_{\mathrm{k}}$.

The competency level in this definition can result from an aggregated evaluation of the $h c_{\mathrm{i}}$ information that is embedded in its $4 C$ elements described in definition 4 . The scale of values for the soft, and even the hard competency levels might be of a qualitative type (e.g. 'low', 'average' or 'high' values), when it is difficult to evaluate such information in a crisp way. Taking this into consideration a corresponding relation hcLevel: $O \times H C \rightarrow[0,100]$ can be defined, which obtains an aggregated competency level value from the information embedded in the $4 C$ elements. In addition, the relation scLevel: $O \times S C \rightarrow\{$ low, average,high $\}$ ) obtains the soft competency level for a given organization. The computation of these levels is further explained in the competencies assessment. A collaboration opportunity $(\mathrm{CO})$ can be related to the competencies that are necessary for its fulfillment. These competencies are usually of the hard type, but the consideration of soft competencies is also necessary. For instance, certain activities associated to a hard competency might require intensive knowledge exchange. In this case, only partners that have such soft competency can assume these activities.

Definition 6 (Collaboration Opportunity Competencies Requirement). Can be specified as a tuple $\mathrm{COCR}=(\mathrm{co}, \mathrm{RC})$ where:

- $c o$ is the identification of a specific collaboration opportunity.

- $R C=\left\{\left(h c_{\mathrm{i}}, s c_{j}, q_{\mathrm{ij}}\right) \mid h c_{\mathrm{i}} \in H C, s c_{\mathrm{j}} \in S C, q_{\mathrm{j}} \in\{\right.$ low,medium,high $\left.\}\right\}$, establishes that an hard competency $h c_{\mathrm{i}}$ requires a certain level $q_{\mathrm{ij}}$ of soft competency $s c_{\mathrm{j}}$ for its performance (e.g. the ability to exchange knowledge).

In addition, we can consider a relation reqLevel: $H C \times S C \times$ $C O \rightarrow\{l o w$, medium, high $\}$, which obtains the soft competency level required by a hard competency needed in a given $C O$, and from the information characterizing that $C O$. The next concept corresponds to the definition of the adjusted competencies model. 
Definition 7 (Organization's adjusted hard Competencies). Represents an organization's adjusted competence profile, in which the competence levels are evaluated, taking into consideration the information provided by the extended model (definition 5 ) and the requirements of a given collaboration opportunity (definition 6). An adjusted competency can be specified as a tuple $\mathrm{ACP}=(o, A H C, c o)$ where

- $o$ is a given organization, such that $o \in O$.

- $c o$ is a specific collaboration opportunity.

- $A H C=\left\{\left\{\left(h c_{\mathrm{i}}, a l_{\mathrm{i}}\right) \mid h c_{\mathrm{i}} \in H C, a l_{\mathrm{i}} \in[0,100]\right\} . a l_{\mathrm{i}}\right.$ represent the adjusted hard competency levels.

The distinction between the extended and the adjusted competences model is that the later is instantiated whenever there is a concrete $C O$ establishing both hard and soft competencies requirements. In such a case, the adjusted model, through its adjusted competency levels, provides better information concerning an organization's effective capacity to use its hard competencies in such collaboration opportunity.

Competencies assessment. The assessment of competencies includes the evaluation of both soft and hard competencies. The information required for the soft competencies evaluation can be obtained from several sources (Fig. 2a), as explained below. These competencies are then used together with the organization's hard competencies and the collaboration opportunity in the calculation of the adjusted hard competency levels (Fig. 2b).
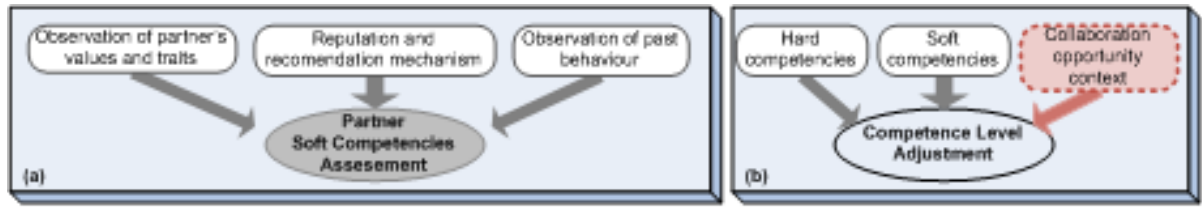

Fig. 2. (a) Soft-Competencies assessment, and (b) hard competencies levels adjustment

The hard competencies of an organization are information that is specified in the provided $4 \mathrm{C}$ elements. The rationality for this assumption is based on the fact that, whenever an organization wishes to join a consortium, it delivers a statement describing its best competencies, aiming at obtaining a favorable qualification.

Soft competencies assessment. For the soft competencies assessment, an adequate way to specify the competency values is required, due to the intrinsic subjectivity and underlying ambiguity that characterizes such type of concepts. For instance, it is not straightforward to provide a percentage value for a soft competency related to the ability to lead a consortium composed of autonomous and conflicting parties. One solution is to adopt a qualitative scale based on:

- Perception of organizations' traits. For instance, the capacity to build consensus on a conflicting situation depends on organization's traits, such as diplomacy and honesty. 
- Receiving advice from a trustworthy partner, who informs about third parties competencies.

- Observation of past behavior, which was characterized as a successful manifestation of a soft competency.

The observation of past behavior is amongst the others the most reliable way to perceive soft competencies. If, for instance, a partner was observed to engage on knowledge sharing on a situation that provided positive outcomes, then it is likely that this partner is willing to engage in such a behavior again in the future. However, if the outcomes were not satisfactory, the partner might be less prone to repeat that behavior. The information concerning past behavior can be obtained, for instance, from a history repository of a $V B E$.

Definition 8 (Soft competencies assessment process). The process that takes an organization's traits, recommendations and observed behavior, in order to infer a level for a given soft competency. This process can be represented as:

$$
\forall_{o} \forall_{s c}(\text { obsern }(o, s c, L) \vee \operatorname{recommend}(o, s c, L) \vee \operatorname{traits}(o, s c, L) \Rightarrow \operatorname{scLevel}(o, s c, L))
$$

The order in which each predicate appears is intended to model the fact that advisor's recommendations are more important than traits perception, and that observed behavior overlaps both recommendations and traits.

Hard competencies levels adjustment. As illustrated in Fig 2b, the hard competency levels adjustment takes as inputs the original soft and hard competency levels (from definition 5), and the requirements of a given $\mathrm{CO}$, including its context, in order to obtain adjusted values for such levels, which provide a more accurate information about the partners performance for the actual CO. It is worth to mention the context, because there might be requirements that are exogenous to the collaboration opportunity (e.g., the necessity to deal with adverse market or to establish relationships with difficult partners).

Definition 9 (Adjusted hard Competency Level). Represents a value obtained with a function adjLevel: $\mathrm{O} \times H C \rightarrow[0,100]$, which for a given organization $o_{\mathrm{i}}$, calculates the adjusted level for its competency $h c_{\mathrm{j}}$, according to the soft competencies owned by the organization, and the ones required by a given collaboration opportunity co. The adjustment function can be specified as:

$$
\operatorname{adjLevel}(o, h c, c o)=h \operatorname{Level}(o, h c) \times \underbrace{\frac{1}{\#\left(S C_{c o, h c}\right)} \times \sum_{n=1}^{\#\left(S C_{c o, h c}\right)}\left[\frac{\operatorname{scLevel}\left(o, s c_{n}\right)}{\operatorname{reqLevel}\left(\operatorname{co}, s c_{n}, h c\right)}\right]}_{\text {Original level }}
$$

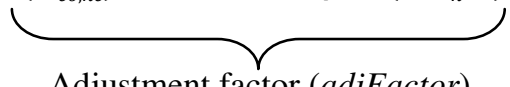

where:

- hcLevel, gives the original organization's hard competency level.

- $o=o_{\mathrm{i}}$ and $h c=h c_{\mathrm{j}}$ for a better function understanding. 
- $\mathrm{SC}_{\mathrm{hc}, \mathrm{co}}$ corresponds to the set of soft competencies that are required in the performance of hard competency $h c$, in the context of the actual $C O$. The expression $\#\left(\mathrm{SC}_{\mathrm{hc}, \mathrm{co}}\right)$ represents the size of this set.

The above expression that computes the adjustment factor is enough for providing the desired adjustment effect. But other expressions reflecting the situations' specificities could also be used. Nonetheless, this adjLevel function requires more generality in order to cope with known cases of collaborative networks. Let us see how this can be done considering the following collaboration-related soft competencies, namely the ability to: (1) perform tasks in a collaborative way, (2) share resources and knowledge, (3) lead a group of autonomous organizations, possibly with conflicting interests and goals, and (4) achieve consensus-based decision-making.

If we consider a single enterprise operating in the market, in which its interactions are mostly transactional, all that matters are hard competencies, with little consideration for collaboration-related soft competencies. This can be modeled as:

$$
\left.\#\left(S C_{c o, h c}\right)\right)=0 \quad \Rightarrow \quad \operatorname{adjLevel}(\mathrm{o}, \mathrm{hc}, \mathrm{co})=\operatorname{hcLevel}(\mathrm{o}, \mathrm{hc})
$$

In an Extended Enterprises (EE) most of the business interactions are controlled by a dominant partner [13]. Nevertheless, the involved entities are autonomous, which requires a greater level of multilateral coordination. A VE/VO, in turn, is essentially constituted by autonomous organizations, which make decisions on a consensus basis [14], and the interactions are mostly collaborative. In this case, there is a higher need for collaboration-related soft competencies. The adjustment level for these two cases can be modeled as:

$$
\left.\#\left(S C_{c o, h c}\right)\right)>0 \quad \Rightarrow \quad \operatorname{adjLevel}(\mathrm{o}, \mathrm{hc}, \mathrm{co})=\mathrm{hcLevel}(\mathrm{o}, \mathrm{hc}) \times \text { adjFactor }
$$

In the case that an organization is invited to join a Virtual Organizations Breeding Environments (VBE), there is no concrete collaboration opportunity to fulfill, neither hard competences requirements to consider at that time. Here, the focus is predominantly put on the organization's soft competencies, being the main concern to assess its preparedness to participate in future partnerships. This can be modeled as:

$$
\left.\#\left(H C_{c o}\right)\right)=0 \quad \Rightarrow \quad \operatorname{adjLevel}\left(o, n i l^{2}, n i l\right)=\text { adjFactor }
$$

\begin{tabular}{|c|c|c|}
\hline $\begin{array}{l}\text { Enterprise in } \\
\text { the marke }\end{array}$ & $\begin{array}{l}\text { Extended } \\
\text { Enterprise }\end{array}$ & VBE \\
\hline $\begin{array}{l}\#\left(S C_{r e q}\right)=0 \\
\#\left(H C_{r e q}\right)>0\end{array}$ & Increase in soft competences importance & $\begin{array}{l}\#\left(S C_{\text {req }}\right)>0 \\
\#\left(H C_{\text {req }}\right)=0\end{array}$ \\
\hline
\end{tabular}

These cases can be classified in a scale, in which they are ordered by their growing needs for soft competences, as illustrated in Fig. 3.

Fig. 3. Soft Competencies importance according to network type

\footnotetext{
${ }^{2}$ Nil corresponds to a non specified value.
} 
Considering these cases, the resulting expression for the adjusted level calculation is given by:

$$
\operatorname{adjLevel}(o, h c, c o)=\left\{\begin{array}{cl}
\text { hcLevel }(o, h c) \times \text { adjFactor } & \Leftarrow \#\left(S C_{c o, h c}\right)>0 \\
h c \text { Level }(o, h c) & \Leftarrow \#\left(S C_{c o, h c}\right)=0 \\
\text { adjFactor } & \Leftarrow \#\left(H C_{c o}\right)=0
\end{array}\right.
$$

An example in next section illustrates how to use this concept.

\section{Modeling Example}

\section{A Prolog implementation}

In order to do some tests and verify the model and its usability, a translation of the above definitions into Prolog rules was made as illustrated in the diagram of Fig.4.

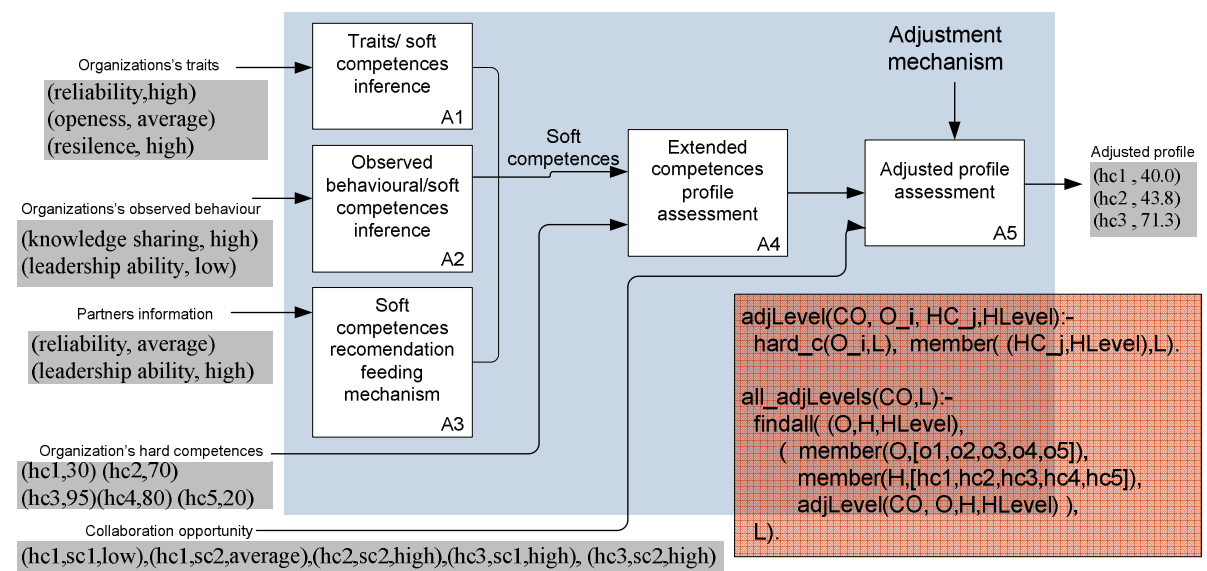

Fig. 4. Diagram for the Prolog version of the competences model

Each block in this diagram represents the assessment steps previously described. Typical inputs and outputs, instantiated from the above definitions are also illustrated.

\section{An illustrative example}

Let us consider a situation in which there is a possibility to engage in a collaboration opportunity. For this opportunity, a set of candidates are considered for the formation of a consortium. This $\mathrm{CO}$ is described in terms of required soft and hard competences, as illustrated in table 1. The values in this table are assumed to be obtained from the identification and characterization of the $\mathrm{CO}$ and its context. By context, we mean the exogenous factors that also imply soft requirements. For instance, this table states that performing $h c_{1}$ is not very demanding on knowledge or resources sharing $\left(s c_{1}, s c_{2}\right)$, that $h c_{2}$ requires a partner with strong leadership quality $\left(s c_{3}\right)$, and that $h c_{3}$ is a competency that requires both intensive knowledge and resources sharing. 
Table 1. Requirements for a collaboration opportunity (L:low, A:average, H:high)

\begin{tabular}{|c|c|c|c|}
\hline \multicolumn{4}{|c|}{ Collaboration opportunity context } \\
\hline \multirow{2}{*}{$\begin{array}{c}\text { Required hard } \\
\text { competencies }\end{array}$} & \multicolumn{3}{|c|}{ Associated (and required)soft competencies } \\
\cline { 2 - 4 } & $\boldsymbol{s c _ { 1 }}$ & $\boldsymbol{s c _ { 2 }}$ & $\boldsymbol{s c _ { 3 }}$ \\
\hline$h c_{1}$ & $\mathrm{~L}$ & $\mathrm{~A}$ & - \\
\hline$h c_{2}$ & - & $\mathrm{H}$ & $\mathrm{H}$ \\
\hline$h c_{3}$ & $\mathrm{H}$ & $\mathrm{H}$ & - \\
\hline
\end{tabular}

A number of organizations have applied to participate in the given CO. Each organization is characterized by its soft and hard competencies, its traits, and its past behaviors, as illustrated in Table 2. In this example, it is established that when we say that a partner assumes a hard competency, it means that it assumes the responsibility to undertake the activities associated to this competency. The soft competencies considered in this example are in the set $S C=\{$ 'ability to share its resources', 'capacity to exchange knowledge', 'ability to lead a consortium' \}. The set considered for traits is $T=\{$ 'reliability', 'adaptability', 'resilience'\}. The soft competency levels are assumed to be obtained as specified in definition 8, more specifically from the organizations' traits and observed behavior.

Table 2. Extended Competency Profile for a set of organizations (Def. 5)

\begin{tabular}{|c|c|c|c|c|c|c|c|c|c|c|c|c|}
\hline & $\begin{array}{l}\text { Observed } \\
\text { Behavior }\end{array}$ & \multicolumn{4}{c|}{ Org. traits } & \multicolumn{1}{c|}{$\begin{array}{l}\text { Soft competen- } \\
\text { cies levels }\end{array}$} & \multicolumn{3}{c|}{ Hard competencies levels } \\
\hline Org. & $(s c$, out $)$ & $t_{1}$ & $t_{2}$ & $t_{3}$ & $s c_{1}$ & $s c_{2}$ & $s c_{3}$ & $h c_{1}$ & $h c_{2}$ & $h c_{3}$ & $h c_{4}$ & $h c_{5}$ \\
\hline $\mathrm{O} 1$ & $\left(\mathrm{sc}_{1}, \mathrm{H}\right),\left(\mathrm{sc}_{3}, \mathrm{~L}\right)$ & $\mathrm{H}$ & $\mathrm{A}$ & $\mathrm{L}$ & $\mathrm{H}$ & $\mathrm{L}$ & $\mathrm{A}$ & 30 & 70 & 95 & 80 & 20 \\
\hline $\mathrm{O} 2$ & $\left(\mathrm{sc}_{2}, \mathrm{H}\right)$ & $\mathrm{H}$ & - & $\mathrm{A}$ & $\mathrm{H}$ & - & $\mathrm{L}$ & 70 & 80 & 50 & 50 & 90 \\
\hline $\mathrm{O} 3$ & $\left(\mathrm{sc}_{1}, \mathrm{H}\right),\left(\mathrm{sc}_{2}, \mathrm{~L}\right)$ & $\mathrm{L}$ & $\mathrm{A}$ & $\mathrm{H}$ & $\mathrm{L}$ & $\mathrm{L}$ & $\mathrm{A}$ & 100 & 50 & 30 & 70 & 70 \\
\hline $\mathrm{O} 4$ & $\left(\mathrm{sc}_{2}, \mathrm{H}\right),\left(\mathrm{sc}_{3}, \mathrm{~L}\right)$ & $\mathrm{H}$ & - & $\mathrm{H}$ & - & - & $\mathrm{H}$ & 80 & 70 & 60 & 30 & 40 \\
\hline $\mathrm{O} 5$ & $\left(\mathrm{sc}_{3}, \mathrm{H}\right)$ & $\mathrm{H}$ & $\mathrm{L}$ & $\mathrm{L}$ & $\mathrm{H}$ & $\mathrm{L}$ & - & 50 & 80 & 30 & 70 & 50 \\
\hline
\end{tabular}

The values for the hard competencies levels were obtained taking in consideration the organizations' competency statements showing the $4 \mathrm{C}$ information (definition 4 and 5), in relation to the critical competencies and resources that are necessary in the collaboration opportunity. The candidates provide that information when they apply for the collaboration opportunity, both to convince that they are qualified, and to be subsequently used in the process planning. The VO planner digests this information and translates it to the aggregated competency levels, illustrated in Table 2, allowing a straightforward comparison between the candidates.

Given the inputs provided in Tables 1 and 2, let us apply the equation specified in definition 9 , in order to determine the adjusted profile of candidate $o_{l}$. Using the referred inputs, the corresponding adjusted levels are:

$$
\begin{aligned}
& \operatorname{adjLevel}\left(\mathrm{o}_{1}, \mathrm{hc}_{1}\right)=30 \% \times 1 / 2 \times(80 / 40+40 / 60)=40 \% \\
& \operatorname{adjLevel}\left(\mathrm{o}_{1}, \mathrm{hc}_{2}\right)=70 \% \times 1 / 2 \times(40 / 80+60 / 80)=44 \% \\
& \operatorname{adjLevel}\left(\mathrm{o}_{1}, \mathrm{hc}_{3}\right)=95 \% \times 1 / 2 \times(80 / 80+40 / 80)=71 \%
\end{aligned}
$$

Performing similarly for the remaining partners, we get the adjusted competency profiles illustrated in Fig. 5, which are next to the initial profiles taken from Table 2. 


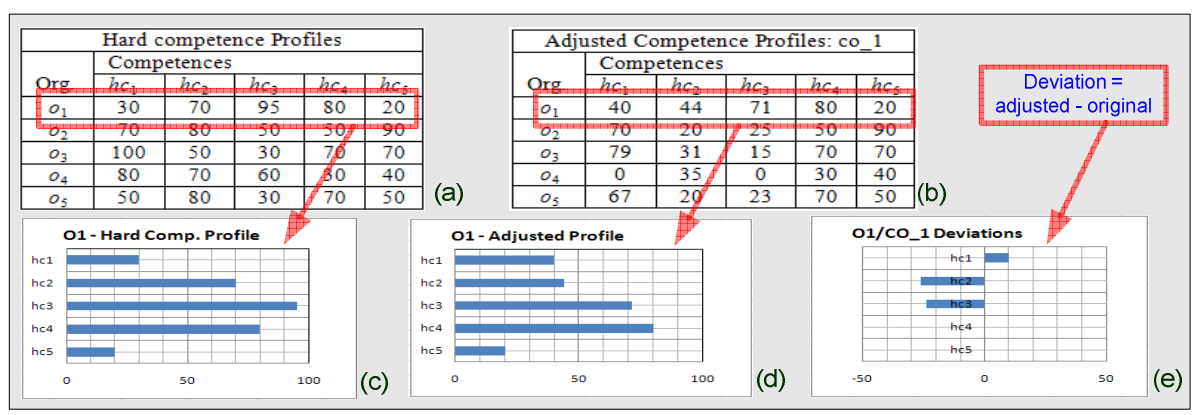

Fig. 5. (a) Original competency levels, and (b) the adjusted values c) $o_{1}$ 's initial profile, (d) the adjusted profile, and (e) the deviations

In order to evaluate partners, it is better to present this information as profile charts, as shown for partner $o_{1}$.

The initial hard competencies profile shows that partner $o_{1}$ would be very competent at performing $h c_{3}$. However, the information in Table 2 states that this partner is low scored at sharing knowledge. But the $C O$ establishes that $h c_{3}$ requires intensive knowledge sharing (table 1). This means that, although being initially considered highly qualified in $h c_{3}$, this partner might in fact display poorer performance in the actual $\mathrm{CO}$, due to its knowledge protection concerns. This fact is illustrated in Fig. 5d, which shows its adjusted profile stating a lower adjusted level for $h c_{3}$, incorporating the knowledge sharing concern. The values that are shown in Fig. 6e correspond to the difference between the adjusted and the original competency levels. Taking in consideration these deviations, this chart shows precisely that there is an inadequate (or negative) adjustment of this partner to the actual $\mathrm{CO}$ in terms of $h c_{3}$, caused by its concern to protect its knowledge. It also suggests that this partner should instead assume the competency $h c_{1}$, which might contradict the initial assumption that this partner was very good on $h c_{3}$, probably the very reason it was initially considered for the consortium. If nevertheless it is decided that partner $o_{1}$ will assume this competence, the remaining partners involved in the consortium should expect problematic interactions with this partner.

\section{Conclusions}

We have addressed the effects of the soft competencies on the performance of the hard ones. An extended partners' competencies abstract model, combining both type of competences was proposed. This model allows the adjustment of the hard competences levels, taking in consideration both soft and hard competencies required in a collaboration opportunity. These dynamically adjusted levels display a more accurate indication about the partners' performance of their competences in the given collaboration opportunities.

The Prolog implementation of the model, together with the illustrative example, shows this approach is useful and that its utilization is straightforward. This approach 
can be easily combined with existing competency models. Furthermore, its integration in a Decision Support System is not a complicated task.

\section{Acknowledgements}

This work was supported in part by the Portuguese "Fundação para a Ciência e a Tecnologia" through two PhD scholarships.

\section{References}

1. McClelland, D.: Testing for competence rather than for intelligence. American Psychologist 20, 321-333 (1973)

2. Dainty, A., Cheng, M.I., Moore, D.R.: A comparison of the behavioural competencies of client-focused and production-focused project managers in the construction sector. Project Management Journal 36, 39-48 (2005)

3. Dubois, D.: Competency-Based Performance: A Strategy for Organizational Change. HRD Press, Boston (1993)

4. Prahalad, C.K., Hamel, G.: The core competence of the corporation. Harvard Business Review 68, 79-90 (1990)

5. Javidan, M.: Core Competence: What does it mean in practice? Long Range Planning 31, 60-71 (1998)

6. Molina, A., Flores, M.: A Virtual Enterprise in Mexico: From Concepts to Practice. Journal of Intelligent and Robotics Systems 26, 289-302 (1999)

7. Boucher, X., Lebureau, E.: Coordination of Competencies Development within Networks of SMEs Collaborative Networks and Their Breeding Environments, vol. 186, pp. 57-66. Springer, Boston (2005)

8. Odenthal, B., Peters, M.: Competence Profiling in Virtual Companies. In: Network-Centric Collaboration and Supporting Frameworks, vol. 224, pp. 143-150. Springer, Boston (2006)

9. Ermilova, E., Afsarmanesh, H.: Competency Modeling Targeted on Promotion of Organizations Towards VO Involvement.: Pervasive Collaborartive Networks, pp. 3-14. Springer, Boston (2008)

10. Soanes, C., Stevenson, A. (eds.): Oxford Dictionary of English, Oxford, UK (2003)

11. Hall, B.: Values Shift: A Guide to Personal and Organizational Transformation. Twin Lights Publishers Rockport, MA (1995)

12. Macedo, P., Sapateiro, C., Filipe, J.: Distinct Approaches to Value Systems in Collaborative Networks Environments. In: Network-Centric Collaboration and Supporting Frameworks, vol. 224, pp. 111-120. Springer, Boston (2006)

13. Camarinha-Matos, L., Afsarmanesh, H.: Collaborative Networks -Value creation in a knowledge society. In: PROLAMAT 2006. Springer, Shanghai (2006)

14. Afsarmanesh, H., Camarinha-Matos, L.M., Ollus, M.: Ecolead and CNO Base Concepts. In: Methods and Tools for Collaborative Networked Organizations, pp. 4-31. Springer, Heidelberg (2008) 